\title{
Functionalization of $\mathrm{BN}$ honeycomb structure by adsorption and substitution of foreign atoms
}

\author{
C. Ataca and S. Ciraci* \\ Department of Physics, Bilkent University, Ankara 06800, Turkey \\ and UNAM-Institute of Materials Science and Nanotechnology, Bilkent University, Ankara 06800, Turkey
}

(Received 4 January 2010; revised manuscript received 3 September 2010; published 1 October 2010)

\begin{abstract}
We carried out first-principles calculations within density-functional theory to investigate the structural, electronic, and magnetic properties of boron-nitride $(\mathrm{BN})$ honeycomb structure functionalized by adatom adsorption, as well as by the substitution of foreign atoms for $\mathrm{B}$ and $\mathrm{N}$ atoms. For periodic high-density coverage, most of $3 d$ transition metal atoms and some of group 3A, 4A, and 6A elements are adsorbed with significant binding energy and modify the electronic structure of bare BN monolayer. While bare BN monolayer is nonmagnetic, wide band-gap semiconductor, at high coverage of specific adatoms it can achieve magnetic metallic, even half-metallic ground states. At low coverage, the bands associated with adsorbed atoms are flat and the band structure of parent BN is not affected significantly. Therefore, adatoms and substitution of foreign atoms at low coverage are taken to be the representative of impurity atoms yielding localized states in the band gap and resonance states in the band continua. Notably, the substitution of C for B and $\mathrm{N}$ yield donorlike and acceptorlike magnetic states in the band gap. Localized impurity states occurring in the gap give rise to interesting properties for electronic and optical application of the single-layer BN honeycomb structure.
\end{abstract}

DOI: 10.1103/PhysRevB.82.165402

PACS number(s): 73.22.-f, 73.90.+f, 75.50.Pp, 75.75.-c

\section{INTRODUCTION}

Research on BN-based materials have grown gradually in recent years. ${ }^{1-5}$ This is not only due to their fascinating properties, such as hardness, high melting point, and large band gap, but also due to the geometric similarity of planar, twodimensional (2D) BN to graphene. Scientists already achieved the synthesis of single-layer BN honeycomb structure on substrates ${ }^{6,7}$ and a few layer thick structures from three-dimensional (3D) hexagonal $\mathrm{BN}$ either on a substrate or freestanding. ${ }^{8-10}$ Recently, Jin et al. ${ }^{11}$ reported the fabrication of freestanding $\mathrm{BN}$ honeycomb structure (we specify it simply as $2 \mathrm{D}$ BN throughout the text). The realization of the synthesis of $2 \mathrm{D} \mathrm{BN}$ is rapidly attracting interest on $\mathrm{BN}$, since it has $2 \mathrm{D}$ hexagonal lattice, which is commensurate to the lattice structure of covalently bonded graphene. More recently, the synthesis of single-layer composite structures consisting of adjacent $2 \mathrm{D} \mathrm{BN}$ and graphene domains is realized. ${ }^{12}$ However, unlike semimetallic graphene, $2 \mathrm{D} \mathrm{BN}$ is a nonmagnetic, wide band-gap semiconductor with an indirect energy gap of $4.64 \mathrm{eV}$ (Ref. 13) calculated within generalized gradient approximation (GGA). The indirect gap is further corrected to $6.82 \mathrm{eV}$ with $\mathrm{GW}_{0}$ self-energy method by Şahin et al. ${ }^{14}$ A theoretical comparative study of 3D and 2D BN, and its nanoribbons comprising their mechanical, electronic, and magnetic properties was reported by Topsakal et $a l .{ }^{13} 2 \mathrm{D} \mathrm{BN}$, and their nanoribbons can be easily functionalized by many different ways for different purposes such as doping, ${ }^{15-17}$ exchange of atoms and vacancies. ${ }^{18,19}$

In this paper, using state-of-the-art first-principles planewave calculations we investigate the effects of adatoms adsorbed on $2 \mathrm{D} \mathrm{BN}$, as well as the substitution of foreign atoms for $\mathrm{B}$ and $\mathrm{N}$ atoms in the honeycomb structure. We consider both high coverage (where the coupling between adjacent foreign atoms is substantial) and low coverage (where the coupling is negligible). We conclude that at high coverage (or decoration) of specific adatoms one can turn the nonmagnetic, wide band-gap material into magnetic, metallic, or even half-metallic states. At low coverage, adatoms give rise to localized states in the band gap.

\section{METHOD}

We perform first-principles, spin-polarized plane-wave calculations $^{20,21}$ within density-functional theory ${ }^{22}$ using projector-augmented-wave potentials. $^{23}$ The exchangecorrelation potential is approximated by GGA. ${ }^{24}$ For the partial occupancies, we use the Methfessel-Paxton smearing method. ${ }^{25}$ The width of the smearing for all structures is chosen as $0.01 \mathrm{eV}$ for geometry relaxations and bandstructure calculations. For accurate density-of-states (DOS) calculations the width of smearing is taken as $0.1 \mathrm{eV}$. We consider a single adatom adsorbed to each $(2 \times 2)$ and $(4 \times 4)$ supercells of $2 \mathrm{D} \mathrm{BN}$ structure and treat the system using periodic boundary conditions. For high coverage corresponding to $\Theta=1 / 8,(2 \times 2)$ supercell is used, while low coverage, $\Theta=1 / 32$, is treated by using $(4 \times 4)$ supercell. A large spacing (at least $\sim 14 \AA$ ) between adjacent $2 \mathrm{D} \mathrm{BN}$ layers is taken to prevent interlayer interactions. The number of plane waves used in expanding Bloch functions and $\mathbf{k}$ points used in sampling the Brillouin zone (BZ) are determined by a series of convergence tests. In the self-consistent potential and the total energy calculations, the BZ is sampled by $(15 \times 15 \times 1)$ mesh points in $\mathbf{k}$ space within MonkhorstPack scheme $\mathrm{e}^{26}$ for the $(2 \times 2)$ supercells. For calculations involving $(4 \times 4)$ supercells, the number of $\mathbf{k}$ points is taken as $(9 \times 9 \times 1)$. For accurate DOS calculations, k-points samplings are further increased to $(25 \times 25 \times 1)$ and $(15 \times 15$ $\times 1)$ for the $(2 \times 2)$ and $(4 \times 4)$ supercells, respectively. A plane-wave-basis set with the kinetic energy cutoff $\hbar^{2} \mid \mathbf{k}$ $+\left.\mathbf{G}\right|^{2} / 2 m=520 \mathrm{eV}$ is used. All the atomic positions and lattice constants are optimized by using the conjugate gradient method, where the total energy and the atomic forces are minimized. The convergence is achieved when the difference 


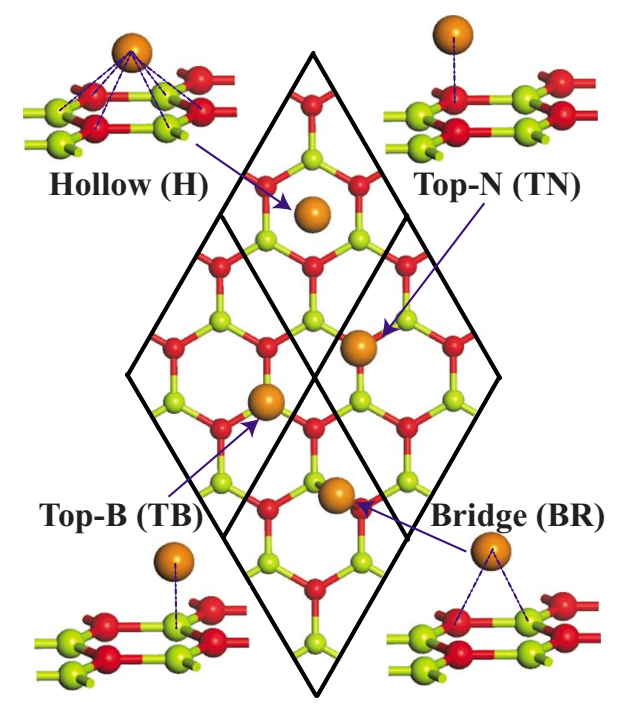

FIG. 1. (Color online) A $(4 \times 4)$ supercell of 2D BN honeycomb structure, which consists of four $(2 \times 2)$ supercells. Various possible adsorption sites of adatoms are indicated. The hollow site, $\mathrm{H}$ : the adatom (gray/orange ball) is placed on top of the center of a hexagon. TN site: the adatom is placed on top of nitrogen (dark gray/ red). TB site: the adatom is placed on top of boron (light gray/ green). The bridge site BR: the adatom is located on top of the boron-nitrogen bond.

of the total energies of last two consecutive steps is less than $10^{-5} \mathrm{eV}$ and the maximum force allowed on each atom is $0.03 \mathrm{eV} / \AA$. The pressure on the system is kept smaller than $\sim 1$ kBar per unit cell in all of the calculations. As a rule, the structure becomes more energetic as its total energy is lowered. Charge-transfer values are calculated according to the Bader analysis. ${ }^{27}$

\section{ADSORPTION}

The lowest energy sites of various adsorbed atoms are determined by placing foreign atoms initially to four possible adsorption sites at a height of $\sim 2 \AA$ from BN plane as described in Fig. 1. Upon fully self-consistent geometry optimizations with both spin-polarized and spin-unpolarized configurations, where all atoms in the supercell are relaxed in all directions, we determine the equilibrium site as the lowest energy configuration among four different sites. The binding energy of an adsorbed adatom is defined as $E_{b}$ $=E_{\mathrm{BN}}+E_{A}-E_{\mathrm{BN}+A}$, where $E_{\mathrm{BN}}$ is the total energy of bare 2D $\mathrm{BN}, E_{A}$ is the total energy of free adatom calculated in the vacuum. $E_{\mathrm{BN}+A}$ is the total energy of $2 \mathrm{D}$ BN structure with adsorbed adatom. We investigated the adsorption of following single adatoms, namely $\mathrm{Sc}, \mathrm{Ti}, \mathrm{V}, \mathrm{Cr}, \mathrm{Mn}, \mathrm{Fe}, \mathrm{Mo}, \mathrm{W}, \mathrm{Pt}$, $\mathrm{H}, \mathrm{C}, \mathrm{Si}, \mathrm{B}, \mathrm{N}, \mathrm{O}, \mathrm{Ca}, \mathrm{Cu}, \mathrm{Pd}, \mathrm{Ni}$, and $\mathrm{Zn}$. Among these atoms, $\mathrm{Cr}, \mathrm{Mn}, \mathrm{Mo}, \mathrm{W}, \mathrm{H}, \mathrm{N}, \mathrm{Ca}$, and $\mathrm{Zn}$ cannot bind to $2 \mathrm{D}$ BN.

\section{A. Adsorption of adatoms to $(2 \times 2) \mathrm{BN}$ supercell $(\Theta=1 / 8)$}

The electronic and magnetic properties of $2 \mathrm{D} B N$ are modified through adatom adsorption at high coverage. The adatom-adatom distance is $\sim 5 \AA$ at $\Theta=1 / 8$, where the magnetic coupling may be crucial in determining the ground state. In order to account for the antiferromagnetic (AFM) coupling between adatoms and to allow their reconstruction we treated $\Theta=1 / 8$ coverage in $(4 \times 4)$ supercell, which includes four $(2 \times 2)$ supercells each having a single adatom. This way adatom-adatom distance of $\sim 5 \AA$ is maintained. We further carried out geometry relaxation with three different initial magnetic ordering of adatoms. First case is where adatoms are coupled antiferromagnetically. The second case is similar to first one but adatoms are initially coupled ferromagnetically (FM). The last case corresponds to a spinunpolarized, nonmagnetic (NM), geometry relaxation. Our calculations indicate that $\mathrm{Cu}, \mathrm{Ni}, \mathrm{Pd}$, and $\mathrm{Pt}$ adatoms have nonmagnetic ground state for $\Theta=1 / 8$, whereas $\mathrm{Sc}$, Ti, and V have AFM ground state. Oxygen is the only adatom which is found to be in the FM ground state. Boron and carbon adatoms undergo a reconstruction to lower the total energy in $(4 \times 4)$ supercell at $\Theta=1 / 8$. Among all adatoms, $\mathrm{Si}$ and $\mathrm{Fe}$ present the most interesting situation, where $2 \mathrm{D} \mathrm{BN}$ monolayer is changed to half-metallic state. However, these adatoms have small binding energies and thus are excluded from our further analysis. Also $\mathrm{Cu}$ having a binding energy smaller than $0.25 \mathrm{eV}$ is also excluded from our study. Our results are given in Table I. In Fig. 2, the calculated energy band structures and corresponding total density of states (TDOS) and partial density of states (PDOS) of $\mathrm{Ni}, \mathrm{Pd}$, or $\mathrm{Pt}$ adatom $+2 \mathrm{D}$ BN system are presented. The band structure of bare $2 \mathrm{D} \mathrm{BN}$ folded to $(2 \times 2) \mathrm{BZ}$ is also presented to reveal the effect of the adatom adsorption on the electronic structure.

Nickel atom with $4 s^{2}+3 d^{8}$ electronic configuration is adsorbed at TN site. Even if $\mathrm{Ni}$ atom is placed at $\mathrm{H}$ or BR sites, it eventually moves to TN site. Flat bands slightly below the Fermi energy are all derived from the $3 d$ orbitals of $\mathrm{Ni}$ atom. The band above the Fermi level is mainly formed from the combination of $4 s$ and $3 d_{z^{2}}$ orbitals of Ni. Accordingly, the band structure of parent $\mathrm{BN}$ layer is not distorted considerably. The charge transfer between $\mathrm{Ni}$ and 2D BN is relatively small and does not cause any significant dipole moment.

Adsorption of Pd and Pt give rise to electronic and magnetic properties similar to those of adsorbed Ni. They are adsorbed also at TN site and have high binding energies. Since the radius of $\mathrm{Pd}$ and $\mathrm{Pt}$ are relatively larger than $\mathrm{Ni}$, the distance to the nearest $\mathrm{N}$ atom of $2 \mathrm{D} \mathrm{BN}, d_{N}$ is slightly larger. The charge transfer between $2 \mathrm{D} \mathrm{BN}$ and the adatom is small. Ni, Pd, and Pt have ionization energies of $7.63 \mathrm{eV}$, $8.33 \mathrm{eV}$, and $8.96 \mathrm{eV}$, respectively. ${ }^{28}$ This ordering of the ionization energies complies with the ordering of work function, $\Phi$, of Ni, Pd, and Pt covered 2D BN. For both Pd and $\mathrm{Pt}$, the bands slightly below the Fermi level are all derived from localized $d$ orbitals but the band above Fermi level is mainly formed from $s$ orbital of adatom with some $d_{z^{2}}$ contribution similar with the case in $\mathrm{Ni}$ adsorption. Even if $\mathrm{Pd}$ and $\mathrm{Pt}$ have the same indirect band gap energy, $\mathrm{Pd}$ shows more dispersive band slightly above the Fermi level.

TDOS and PDOS corresponding to $\Theta=1 / 8$ coverage of $\mathrm{B}, \mathrm{C}$ [which undergo a reconstruction in $(4 \times 4)$ supercell]; $\mathrm{Sc}, \mathrm{Ti}, \mathrm{V}$ (which have AFM ground state), and $\mathrm{O}$ (which has FM ground state) are presented in Fig. 3. Four carbon 
TABLE I. Calculated structural, electronic, and magnetic properties of $2 \mathrm{D} \mathrm{BN}$ monolayer at uniform $\Theta=1 / 8$ adatom coverage. Equilibrium positions of the adatoms, such as TN, TB, H, and BR are described in Fig. 1; distances of adatom to the nearest N, $d_{N}$ (in $\AA$ ); distances of adatom to the nearest $\mathrm{B}, d_{B}$ (in $\AA$ ); the height of adatom from the BN plane, $h$ (in $\AA$ ); the average $\mathrm{B}-\mathrm{N}$ bond length, $d_{\mathrm{BN}}$ (in $\AA$ ); the binding energy of adatom, $E_{b}$ (in eV); and the net magnetic moment per supercell, $\mu$ (in $\mu_{B}$ ). Electronic structure is specified as metallic (M) or semiconductor (SC). The type of the band gap can be either direct $(d r)$ or indirect gap (id). The energy gap of the system after adsorption, $E_{g}$ (in $\mathrm{eV}$ ); the transfer of charge to the adatom from $\mathrm{BN}, \Delta \rho$ (in electrons and $\Delta \rho<0$, if adatom is negatively charged); the dipole moment of the system along the $z$-direction, $p$ (in $e \AA$ ); and work function (or photoelectric threshold for semiconductors) is $\Phi$ (in $\mathrm{eV})$. For the adatoms, which have either NM or FM ground states, calculations are performed using $(2 \times 2)$ supercell; whereas for adatoms, which have either AFM ground state or reconstruction, $(4 \times 4)$ supercell are used with $\Theta=1 / 8$. For adatoms undergoing a reconstruction, $\Delta \rho$ is given for the average charge transfer per adatom.

\begin{tabular}{|c|c|c|c|c|c|c|c|c|c|c|c|c|c|c|}
\hline Magnetic ground state & Adatom & Position & $d_{N}$ & $d_{B}$ & $h$ & $d_{\mathrm{BN}}$ & $E_{b}$ & $\mu$ & Electronic structure & Gap type & $E_{g}$ & $\Delta \rho$ & $p$ & $\Phi$ \\
\hline \multirow[t]{3}{*}{ NM } & $\mathrm{Ni}$ & $\mathrm{TN}$ & 1.87 & 2.31 & 1.84 & 1.46 & 1.14 & & SC & $d r$ & 0.43 & 0.17 & -0.18 & 3.91 \\
\hline & $\mathrm{Pd}$ & $\mathrm{TN}$ & 2.15 & 2.57 & 2.12 & 1.46 & 0.94 & & $\mathrm{SC}$ & $i d$ & 0.93 & 0.02 & -0.15 & 4.41 \\
\hline & $\mathrm{Pt}$ & $\mathrm{TN}$ & 2.04 & 2.49 & 2.04 & 1.46 & 1.48 & & $\mathrm{SC}$ & $i d$ & 0.93 & -0.03 & -0.10 & 4.66 \\
\hline FM & $\mathrm{O}$ & TB & 2.33 & 1.47 & 1.76 & 1.46 & 0.98 & 2.00 & $\mathrm{SC}$ & $i d$ & 0.05 & -0.64 & 0.24 & 7.02 \\
\hline \multirow[t]{3}{*}{ AFM } & $\mathrm{Sc}$ & $\mathrm{H}$ & 2.49 & 2.54 & 2.03 & 1.46 & 0.90 & 0.00 & M & & & 0.67 & 0.30 & 3.03 \\
\hline & $\mathrm{Ti}$ & $\mathrm{H}$ & 2.44 & 2.48 & 1.99 & 1.45 & 0.92 & 0.00 & M & & & 0.52 & 0.98 & 3.56 \\
\hline & V & $\mathrm{TN}$ & 2.21 & 2.67 & 2.26 & 1.45 & 0.60 & 0.00 & $\mathrm{SC}$ & $i d$ & 0.19 & 0.36 & 0.04 & 2.81 \\
\hline \multirow[t]{2}{*}{ NM (Reconstruction) } & B & $\mathrm{TN}$ & 1.61 & 1.98 & 1.58 & 1.46 & 0.80 & & $\mathrm{SC}$ & $i d$ & 0.64 & 0.40 & 1.09 & 5.45 \\
\hline & $\mathrm{C}$ & $\mathrm{BR}+\mathrm{TN}$ & 1.52 & 1.66 & 1.28 & 1.46 & 1.38 & & $\mathrm{SC}$ & $d r$ & 0.35 & -0.45 & 0.54 & 5.74 \\
\hline
\end{tabular}

adatoms treated in a $(4 \times 4)$ supercell undergo a reconstruction; ${ }^{29}$ while three $\mathrm{C}$ atoms are adsorbed near $\mathrm{TN}$ sites, the remaining one is moved to the BR site. Upon reconstruction, the charge on $\mathrm{C}$ adatom at the bridge cite is minute positive, whereas those at $\mathrm{TN}$ site are negatively charged by $\sim 0.6$. Carbon adatoms at $\mathrm{TN}$ site take their charge mostly from the nearest $\mathrm{N}$ atom. The bonding of $\mathrm{C}$ at $\mathrm{TN}$ cite has an ionic character resulting a dipole moment on the system.

$\mathrm{Sc}$, Ti, and V have AFM ground state at $\Theta=1 / 8 .{ }^{30}$ Unlike $\mathrm{Sc}$ and $\mathrm{Ti}$, adsorption of $\mathrm{V}$ does not make the system metallic. The energy bands below the Fermi level is mainly from
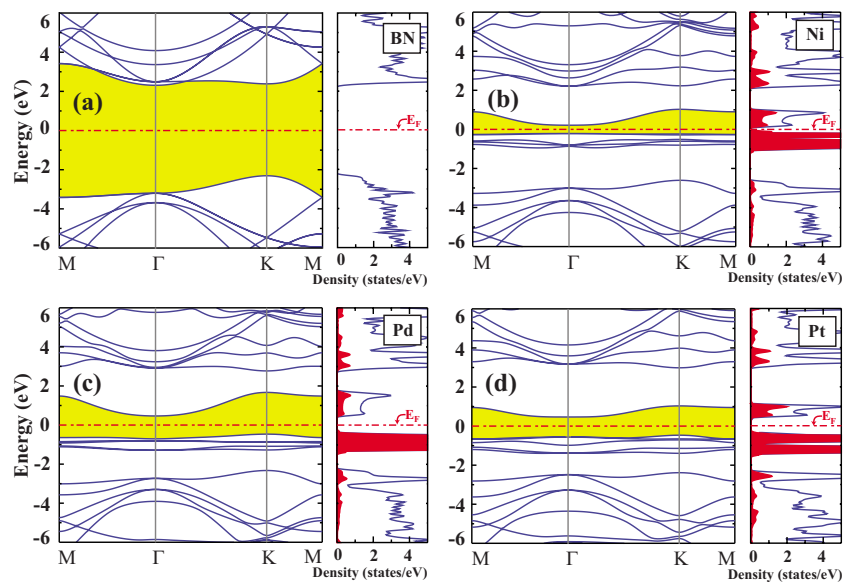

FIG. 2. (Color online) (a) The energy band structure of bare 2D $\mathrm{BN}$ folded to the $(2 \times 2)$ supercell and corresponding TDOS. Zero of band energy is set at the Fermi energy, $E_{F}$. (b)-(d) The energy band structure of single $\mathrm{Ni}, \mathrm{Pd}$, and $\mathrm{Pt}$ adsorbed to each $(2 \times 2)$ supercell of 2D BN $(\Theta=1 / 8)$. Corresponding TDOS (continuous black/blue line) and PDOS projected to the adatom (shaded dark/ red) are also indicated. The energy gap of semiconductors are shaded (light/yellow). The ground states are nonmagnetic. $d_{x y}, d_{y z}, d_{x z}$ and above the Fermi level is mainly from $d_{x^{2}}$ and $d_{z}$. The energy band gap of $0.19 \mathrm{eV}$ originates from the splitting of $3 d$ orbital states.

Among all adatoms in Table I, only $\mathrm{O}$ has FM ground state and is absorbed at TB site. The excess charge on the
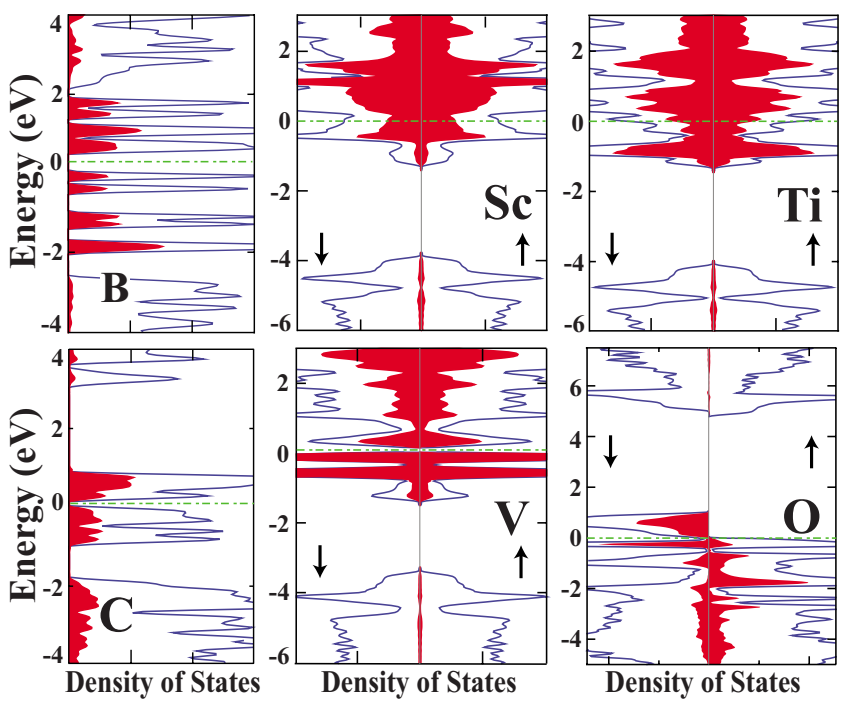

FIG. 3. (Color online) TDOS (dark/blue line) and PDOS (shaded gray/red) for $\mathrm{B}, \mathrm{C}, \mathrm{O}, \mathrm{Sc}, \mathrm{Ti}$, and $\mathrm{V}$ adatoms at high coverage, $\Theta=1 / 8$. Up (down) arrow on the right (left) site of TDOS and PDOS indicates spin direction. Zero of the DOS energy is set to the Fermi energy, $E_{F}$ of the adatom $+2 \mathrm{D}$ BN system which is indicated with dashed-dotted gray/green line. All adatoms treated in $(4 \times 4)$ supercell to allow antiferromagnetic interaction or reconstruction, whereby single adatom is adsorbed to each $(2 \times 2)$ supercells of $(4 \times 4)$ supercell amounting $\Theta=1 / 8$. Sc, Ti, and $\mathrm{V}$ have antiferromagnetic ground state; $\mathrm{O}$ has ferromagnetic state; and $\mathrm{B}$ and $\mathrm{C}$ are nonmagnetic. The state densities are given in arbitrary units but have the same scale for all adatoms. 
TABLE II. Binding energies $\left(E_{b}\right)$, magnetic moments $(\mu)$, adsorption sites, and average adatom-adatom distances (Ref. 31) $\left(\bar{d}_{A-A}\right)$ of C, O, Sc, Ti, and Pt adatoms adsorbed on 2D BN at different coverages.

\begin{tabular}{lcccccc}
\hline \hline$\Theta$ & & $\mathrm{C}$ & $\mathrm{O}$ & $\mathrm{Sc}$ & $\mathrm{Ti}$ & $\mathrm{Pt}$ \\
\hline $1 / 8$ & $E_{b}(\mathrm{eV})$ & 1.38 & 0.98 & 0.90 & 0.92 & 1.48 \\
$\bar{d}_{A-A} \sim 5 \AA$ & $\mu\left(\mu_{B}\right)$ & & 2.00 & 0.00 & 0.00 & \\
$1 / 32$ & Site & $\mathrm{BR}+\mathrm{TN}$ & $\mathrm{TB}$ & $\mathrm{H}$ & $\mathrm{H}$ & $\mathrm{TN}$ \\
& & & & & & \\
$\bar{d}_{A-A}=\sim 10 \AA$ & $E_{b}(\mathrm{eV})$ & 1.17 & 2.01 & 0.43 & 0.74 & 1.52 \\
$1 / 128$ & $\mu\left(\mu_{B}\right)$ & 2.00 & & 3.00 & 4.00 & \\
$\bar{d}_{A-A}=\sim 20 \AA$ & Site & $\mathrm{BR}$ & $\mathrm{BR}$ & $\mathrm{H}$ & $\mathrm{H}$ & $\mathrm{TN}$ \\
\hline \hline
\end{tabular}

adsorbed $\mathrm{O}$ is transferred from three nearest $\mathrm{N}$ atoms, which leads to an ionic character in bonding. Since underlying B atom is positively charged and $\mathrm{O}$ is negatively charged, adsorbed $\mathrm{O}$ pulls the nearest $\mathrm{B}$ atom and causes distortion on planar 2D BN monolayer.

\section{B. Adsorption of adatoms to $(4 \times 4) \mathrm{BN}$ supercell $(\Theta=1 / 32)$}

We next investigate the adsorption of a single adatom to $(4 \times 4)$ supercell, which corresponds to low density coverage, $\Theta=1 / 32$. As indicated in Fig. 1, we initially placed the adatoms at four different positions and relaxed their geometric structure with both spin-polarized and spin-unpolarized calculations. Interestingly, we observe that among nine different adatoms, the adsorption sites of $\mathrm{B}, \mathrm{C}$, and $\mathrm{O}$ differ by going from $\Theta=1 / 8$ to $\Theta=1 / 32$. Since the adatom-adatom distance between adjacent supercells is $\sim 10 \AA$, it can normally be contemplated that the coupling between adatoms are negligible. To verify this assumption, we carried out binding energy calculations for $\mathrm{C}, \mathrm{O}, \mathrm{Ti}, \mathrm{Sc}$, and $\mathrm{Pt}$ adatoms in $(8 \times 8)$ supercell corresponding to a coverage of $\Theta$ $=1 / 128$. In Table II we compared the equilibrium binding sites and binding energies, and magnetic states of these atoms for three different coverage $\Theta=1 / 8,1 / 32$, and $1 / 128$.

The binding energies as well as the magnetic ground states of $\mathrm{C}, \mathrm{O}, \mathrm{Sc}$, and $\mathrm{Ti}$ vary substantially by going from $\Theta=1 / 8$ to $\Theta=1 / 32$. For $\mathrm{C}$ and $\mathrm{O}$, the adsorption sites are also changed. However the situation is not the same when the coverage is further lowered from $\Theta=1 / 32$ to $\Theta=1 / 128$ hence when the adatom-adatom distance is increased from $\sim 10 \AA$ to $\sim 20 \AA$. For adatoms included in Table II, the binding energies and magnetic moments are not changing significantly, and the adsorption sites are remaining the same by going from $\Theta=1 / 32$ to $\Theta=1 / 128$. This finding is corroborating our arguments made at the beginning of the paper, that the properties calculated for $\Theta=1 / 32$ coverage [or the adsorption of a single adatom adsorbed to each $(4 \times 4)$ supercell] can mimic the adsorption of single isolated adatom on a very large area of 2D BN (or very large adatom-adatom distance). Consequently, the bands of adatom $+2 \mathrm{D}$ BN calcu- lated at $\Theta=1 / 32$ become rather flat and can be taken as the localized impurity state (or resonances if $2 \mathrm{D} \mathrm{BN}$ states are significantly contributed). Under these circumstances, the band gap and the edges of valence and conduction bands can be unaltered. In Table III, we include the calculated geometric, electronic and magnetic properties of a single adatom adsorbed to $2 \mathrm{D} B N$ at $\Theta=1 / 32$. The energies of localized and resonance states relative to $2 \mathrm{D} \mathrm{BN}$ 's valence band edge are also tabulated.

Boron adsorbed on $2 \mathrm{D} \mathrm{BN}$ on $(4 \times 4)$ supercell exhibits electronic, magnetic, and structural properties, which are significantly different from those of $\Theta=1 / 8$. For example, $B$ atom of the planar $\mathrm{BN}$ rises $\sim 0.2 \AA$; this slightly changes the $s p^{2}$-hybridization locally. Two spin-up bands and one spin-down band are filled below the Fermi energy. In Fig. 4, the band structure of $\mathrm{B}+2 \mathrm{D} \mathrm{BN}$ and isosurfaces of charge density corresponding to the localized states $\mathrm{E}_{1}, \mathrm{E}_{3}$, and $\mathrm{E}_{5}$ are shown. The magnetic properties are different from the case at $\Theta=1 / 8$ since reconstruction of $B$ adatoms does not take place due to the absence of adatom-adatom interaction.

Adsorption of $\mathrm{C}$ on $(4 \times 4)$ supercell of $2 \mathrm{D} \mathrm{BN}$ causes significant splitting of degenerate $p$ orbital levels. Spin-up and spin-down bands indicated in Fig. 4 originate from $p_{x}$ $\left(\mathrm{E}_{1}\right.$ state $)$ and $p_{y}\left(\mathrm{E}_{2}\right.$ state $)$ orbitals of adsorbed $\mathrm{C}$ atom. In this low coverage, $\mathrm{C}$ adatom is adsorbed to $\mathrm{BR}$ position, but closer to $\mathrm{N}$ atom. Similar to $\mathrm{B}$, the magnetic properties of the $\mathrm{C}+\mathrm{BN}$ system changed from NM to FM upon lowering the coverage density.

At $\Theta=1 / 8, O$ adatom creates a surface distortion and pulls underlying $B$ atom upwards. However at $\Theta=1 / 32, \mathrm{O}$ adsorbed at BR site does not generate a distorted $\mathrm{BN}$ region. The bands near the edge of valence band have significant dispersion due to strong coupling between $\mathrm{O}$ and $2 \mathrm{D} \mathrm{BN}$. The AFM ground states of $\mathrm{Sc}$, Ti, and $\mathrm{V}$ adsorbed $2 \mathrm{D} \mathrm{BN}$ at $\Theta=1 / 8$ change to FM ground states at $\Theta=1 / 32$. Among all adatoms studied in the present paper, electronic and magnetic properties of $\mathrm{Ni}, \mathrm{Pd}$, and $\mathrm{Pt}$ are not affected upon lowering the coverage density.

Next we address the question whether adsorbed adatom migrates on 2D BN honeycomb structure. Here we consider 
TABLE III. Calculated values for single adatom adsorbed to each $(4 \times 4)$ supercell, corresponding to the coverage $\Theta=1 / 32$. Adsorption site, binding energy, $E_{b}$; magnetic moment per $(4 \times 4)$ supercell, $\mu$; the distance from $2 \mathrm{D} \mathrm{BN}$ monolayer, $h$; the distance from the nearest $\mathrm{N}$ atom, $d_{N}$; the distance from the nearest B atom, $d_{B}$; charge transfer, $\Delta \rho$; and energies of relevant localized or resonance states measured from the top of the valence band, $\mathrm{E}_{n}$. $(\mathrm{F} \uparrow)$ indicates that the corresponding spin-up state is full. (E $\downarrow$ ) represents an unoccupied spin-down state. If no spin direction is indicated, that state is nonmagnetic. $\mathrm{R}$ is resonance state having significant contribution from $2 \mathrm{D}$ BN states in the band continua. Adsorption site of the adatoms corresponding to their lowest total energy are indicated by TN, TB, BR, or H as described in Fig. 1.

\begin{tabular}{|c|c|c|c|c|c|c|c|c|c|}
\hline & $\mathrm{Ni}$ & $\mathrm{Pd}$ & $\mathrm{Pt}$ & $\begin{array}{c}\mathrm{C} \\
\mathrm{BR}\end{array}$ & $\mathrm{Sc}$ & $\mathrm{Ti}$ & $\mathrm{V}$ & $\begin{array}{c}\mathrm{B} \\
\mathrm{BR}\end{array}$ & $\begin{array}{c}\mathrm{O} \\
\mathrm{BR}\end{array}$ \\
\hline Site & TN & & & & & & & & \\
\hline $\mathrm{E}_{b}(\mathrm{eV})$ & 1.15 & 0.96 & 1.52 & 1.17 & 0.43 & 0.74 & 0.58 & 0.79 & 2.01 \\
\hline$\mu\left(\mu_{B}\right)$ & & & & 2.00 & 3.00 & 4.00 & 5.00 & 1.00 & \\
\hline$h(\AA)$ & 1.87 & 2.15 & 2.14 & 1.71 & 2.08 & 2.04 & 2.28 & 1.83 & 1.56 \\
\hline$d_{N}(\AA)$ & 1.86 & 2.14 & 2.02 & 1.59 & 2.51 & 2.46 & 2.21 & 1.63 & 1.52 \\
\hline$d_{B}(\AA)$ & 2.31 & 2.56 & 2.50 & 1.77 & 2.53 & 2.49 & 2.66 & 1.79 & 1.48 \\
\hline$\Delta \rho$ & 0.16 & 0.01 & -0.01 & -0.23 & 0.59 & 0.42 & 0.29 & 0.30 & -0.81 \\
\hline $\mathrm{E}_{1}$ & $1.43(\mathrm{~F})$ & $0.65(\mathrm{~F})$ & $0.32(\mathrm{~F})$ & $1.11(\mathrm{~F} \uparrow)$ & $2.72(\mathrm{~F} \uparrow)$ & $2.73(\mathrm{~F} \uparrow)$ & $2.16(\mathrm{~F} \uparrow)$ & $0.26(\mathrm{~F} \uparrow)$ & $-0.21(\mathrm{FR})$ \\
\hline$E_{2}$ & $1.83(\mathrm{~F})$ & $0.92(\mathrm{~F})$ & $0.61(\mathrm{~F})$ & $1.61(\mathrm{~F} \uparrow)$ & $3.14(\mathrm{~F} \uparrow)$ & $3.35(\mathrm{~F} \uparrow)$ & $2.57(\mathrm{~F} \uparrow)$ & $0.70(\mathrm{~F} \downarrow)$ & $-0.12(\mathrm{FR})$ \\
\hline$E_{3}$ & $2.80(\mathrm{E})$ & $1.18(\mathrm{~F})$ & $0.79(\mathrm{~F})$ & $2.39(\mathrm{E} \downarrow)$ & $3.75(\mathrm{E} \downarrow)$ & $3.97(\mathrm{E} \uparrow)$ & $3.01(\mathrm{~F} \uparrow)$ & $2.02(\mathrm{~F} \uparrow)$ & 0.49 (FR) \\
\hline $\mathrm{E}_{4}$ & 4.49 (ER) & $2.95(\mathrm{E})$ & $2.25(\mathrm{E})$ & $3.38(\mathrm{E} \downarrow)$ & $3.77(\mathrm{E} \uparrow)$ & $4.06(\mathrm{E} \downarrow)$ & $3.48(\mathrm{E} \uparrow)$ & $3.02(\mathrm{E} \downarrow)$ & $0.66(\mathrm{FR})$ \\
\hline $\mathrm{E}_{5}$ & & & & & $3.96(\mathrm{E} \downarrow)$ & $4.74(\mathrm{E} \downarrow)$ & $3.88(\mathrm{E} \downarrow)$ & $3.18(\mathrm{E} \downarrow)$ & 4.14 (ER) \\
\hline $\mathrm{E}_{6}$ & & & & & $4.01(\mathrm{E} \uparrow)$ & & $4.32(\mathrm{E} \downarrow)$ & $3.42(\mathrm{E} \uparrow)$ & \\
\hline $\mathrm{E}_{7}$ & & & & & $4.49(\mathrm{E} \downarrow)$ & & $4.77(\mathrm{E} \downarrow)$ & & \\
\hline
\end{tabular}

only single $\mathrm{C}$ and $\mathrm{Ti}$ atoms as prototypes and calculate the energy barrier for their motion along the symmetry directions of 2D BN. In Fig. 5, the variation in energy of single $\mathrm{C}$ and $\mathrm{Ti}$ adatoms along symmetry directions are presented. The potential barrier on the migration path for $\mathrm{C}$ adatom is $\sim 0.30 \mathrm{eV}$. In the case of $\mathrm{Ti}$ the energy barrier on the possible migration path is only $0.15 \mathrm{eV}$. The latter barrier is not high enough to prevent $\mathrm{Ti}$ atoms from cluster formation at elevated temperatures.

\section{SUBSTITUTION $(\Theta=1 / 32)$}

We, finally deal with the substitutional doping of the specific atoms. Here we consider that $\mathrm{Al}, \mathrm{Be}$, and $\mathrm{C}$ substitution for $\mathrm{B}$ atom; $\mathrm{C}, \mathrm{O}$, and $\mathrm{P}$ substitution for $\mathrm{N}$ atom of $2 \mathrm{D} \mathrm{BN}$ honeycomb structure. We mimic the substitutional doping by a model, where a specific atom substitutes for a single $\mathrm{B}$ or $\mathrm{N}$ in every $(4 \times 4)$ supercell of $2 \mathrm{D} \mathrm{BN}$. In this model the distance between impurity atoms is $\sim 10 \AA$ resulting in negligible coupling between them. The substitution energy $E_{s}$ is calculated $^{32,33}$ as

$$
E_{s}=\frac{N-1}{N} E_{\mathrm{BN}}+E_{A}-E_{\mathrm{B} \vee \mathrm{N}}-E_{\mathrm{BNS}}
$$

Here $N$ is the number of atoms in the supercell, which is $N=32$ for $(4 \times 4)$ supercell. $E_{\mathrm{BN}}$ is the calculated total energy of $2 \mathrm{D} \mathrm{BN}$ of the $(4 \times 4)$ supercell corresponding to $16 \mathrm{~B}-\mathrm{N}$ atom pairs. $E_{A}$ is the experimental cohesive energy ${ }^{28}$ of the impurity atom in its equilibrium crystal. $E_{\mathrm{BNS}}$ is the total energy of the supercell after substitution process. $E_{\mathrm{B} \vee \mathrm{N}}$ is the cohesive energy of either $\mathrm{B}$ or $\mathrm{N}$ that depends on which atom is exchanged. The experimental cohesive energy of B is defined with respect to the $\mathrm{B}$ crystal. ${ }^{28}$ However, in the case of $\mathrm{N}$, the cohesive energy of $\mathrm{N}$ is calculated relative to $\mathrm{N}_{2}$ in the gas phase. According to this expression, a positive energy value means the exchange of foreign adatom either with $B$ or $\mathrm{N}$ is endothermic reaction, whereas the negative value indicates an exothermic process. Structural relaxations and the lowest energy states of the final structures are further tested with three different initial magnetic moment distributions on the atoms and all these cases are converged to the same values given in Table IV. Here the substitution of $\mathrm{C}$ atom for $\mathrm{B}(\mathrm{N})$ is of particular interest, since it dopes the $2 \mathrm{D} \mathrm{BN}$ honeycomb structure as donor (acceptor).

We now examine the electronic and magnetic structure of 2D BN substituted by $\mathrm{Al}, \mathrm{C}$, and $\mathrm{P}$ described schematically in Fig. 6. Al substituting for B and being in the same group with $\mathrm{B}$, but having a relatively large atomic radius, distorts the planar structure of BN layer and is located at a position $0.52 \AA$ higher than the substituted $\mathrm{B}$ atom. The valence band of the BN layer does not influence much upon substitution, but states derived from $p$ orbitals of $\mathrm{Al}$ adatom appear near the conduction band edge. In Fig. 6, $p_{z}$ orbitals of $\mathrm{Al}$ are dominant in $\mathrm{E}_{1}$ state, however $p_{y}$ is the most contributed in $\mathrm{E}_{2}$ state. In the case of $\mathrm{C}$ substituting $\mathrm{B}$, the valence and conduction bands of $\mathrm{BN}$ layer are not influenced, except minute splitting between the spin-up and spin-down states resulting from the magnetic $\mathrm{C}$ atom. The excess electron of the substituted $\mathrm{C}$ atom relative to $\mathrm{B}$ leads spin-polarization and fills the $\mathrm{E}_{1}$ state in Fig. 6 like a $n$-type semiconductor. When substitute for $\mathrm{N}, \mathrm{C}$ atom creates electron deficiency; states derived from $p$ orbitals of $\mathrm{C}$ atom are located near the 


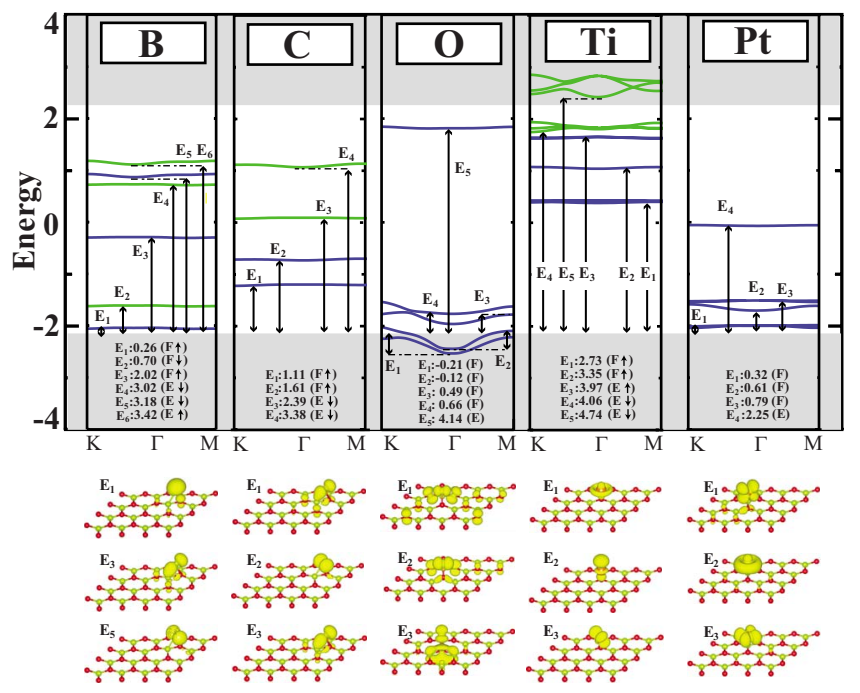

FIG. 4. (Color online) Schematic of the relevant energy levels (or bands) of adatom (B, C, O, Ti, and $\mathrm{Pt}$ ) adsorbed to $(4 \times 4)$ supercell of the single layer $\mathrm{BN}$. The light/gray shaded region in the background is the valence and conduction band region of $2 \mathrm{D} \mathrm{BN}$. Zero of band energy is set at the Fermi level of the parent, bare 2D $\mathrm{BN}, E_{F}$. Spin-up and spin-down bands are shown by dark/blue and light/green lines, respectively. Solid bands indicates that the contribution of adatom to the band is more than $50 \%$ except $\mathrm{O}$ adatom. Energies of some of the relevant adatom (impurity) states relative to valence band edge of parent $2 \mathrm{D} \mathrm{BN}$ are indicated. Below each energy diagram of adatoms, charge density isosurfaces of specific states are shown. The isosurface value is taken as 7 $\times 10^{-5}$ electrons $/ \AA^{3}$. (Note that all localized states of $\mathrm{O}$ have significant contributions from BN states and are shown by solid dark/ blue lines.)

edge of the valence band like a $p$-type semiconductor. $p_{z}$ orbital of $\mathrm{C}$ splits and spin-up ( $\mathrm{E}_{3}$ state in Fig. 6) becomes occupied. Because of this unpaired electron $C$ atom attains net $1 \mu_{B}$ total magnetic moment in both $n$-type and $p$-type substitutional dopings.

Substituted $\mathrm{P}$ having relatively larger atomic radius, gives rise to the local deformation and raises $1.42 \AA$ above the plane of $2 \mathrm{D} \mathrm{BN}$ honeycomb structure. This gives rise to a local dehybridization of planar $s p^{2}$ bonding. Since the upper valence bands of parent 2D $\mathrm{BN}$ are derived mainly from nitrogen atoms, these bands are affected upon their exchange with foreign atoms. Hence several resonances appear, such as $E_{1}$ and $E_{2}$ in the upper part of valence band when $P$ substitutes for $\mathrm{N}$ atom in Fig. 6. In addition a localized state $\mathrm{E}_{3}$ occurs near the conduction band edge.

\section{DISCUSSION AND CONCLUSIONS}

In this paper, we showed that 2D BN can be functionalized to attain properties, which can be useful in future applications in nanoelectronics and nanomagnetics. Functionalization can be achieved either through the adsorption of foreign atoms at different coverage or substitution of foreign atoms for $\mathrm{B}$ or $\mathrm{N}$ in honeycomb structure. We considered a number of foreign atoms, such as $\mathrm{Sc}, \mathrm{Ti}, \mathrm{V}, \mathrm{Cr}, \mathrm{Mn}, \mathrm{Fe}, \mathrm{Mo}$, W, Pt, H, C, Si, B, N, O, Ca, Cu, Pd, Ni, and Zn. Part of
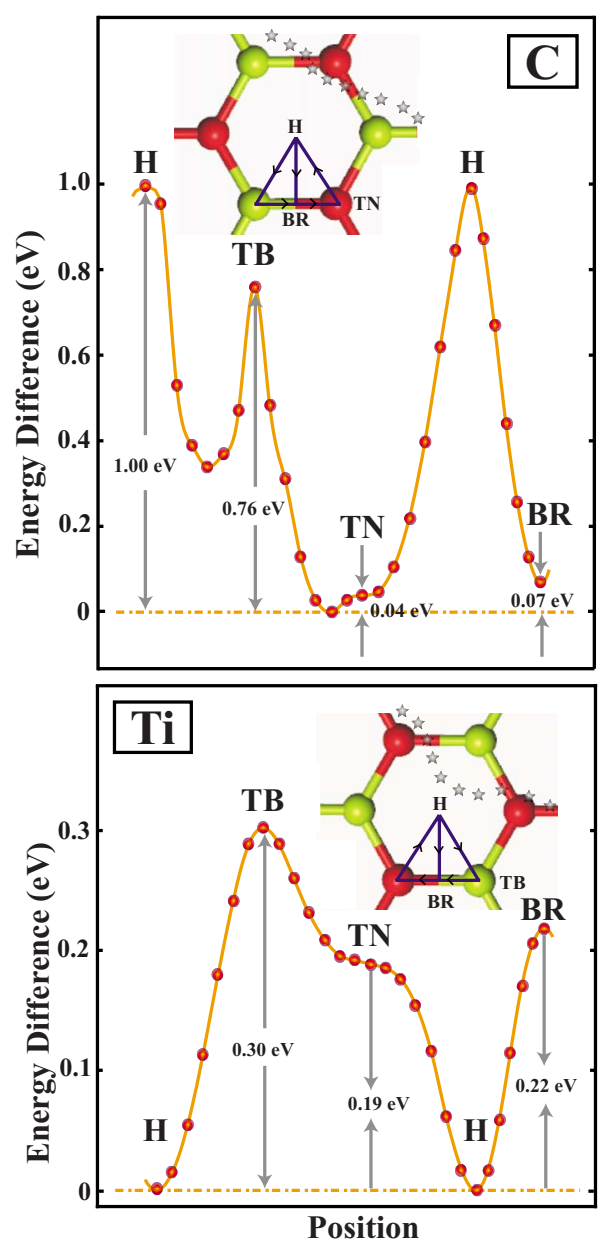

FIG. 5. (Color online) Variation in energy of a single $\mathrm{C}$ and $\mathrm{Ti}$ adatom as they are moving along special directions of a hexagon of 2D BN honeycomb structure. The possible migration path of adatom facing minimum energy barrier is shown by stars on the hexagon. Calculations are carried out in $(4 \times 4)$ supercell.

these atoms are bound with a significant energy and form chemical bonds with 2D BN. $\mathrm{Cu}, \mathrm{Fe}$, and $\mathrm{Si}$ have binding energy smaller than $0.25 \mathrm{eV}$, but $\mathrm{Cr}, \mathrm{Mn}, \mathrm{Mo}, \mathrm{W}, \mathrm{H}, \mathrm{N}, \mathrm{Ca}$, and $\mathrm{Zn}$ cannot bind to $2 \mathrm{D} \mathrm{BN}$. Owing to the van der Waals interaction, the true binding energies can be $0.1-0.2 \mathrm{eV}$ larger than those calculated with GGA in the present work.

High coverage of adatoms corresponding to $\Theta=1 / 8$ leads to dramatic modifications in electronic structure, if the related binding energy is significant. Under these circumstances, either the wide band gap of 2D BN can be reduced or diminished and the system becomes metallic. Under certain circumstances, the nonmagnetic 2D BN attains magnetic moment. $\mathrm{Ni}, \mathrm{Pd}$, and $\mathrm{Pt}$ covered 2D BN are nonmagnetic semiconductors with band gap relatively smaller than that of the parent 2D BN. Sc, Ti forming a $(2 \times 2)$ structure on $2 \mathrm{D}$ $\mathrm{BN}$ are AFM metals, $\mathrm{V}+2 \mathrm{D} \mathrm{BN}$ is an AFM semiconductor. Remarkably, $\mathrm{B}+$ and $\mathrm{C}+2 \mathrm{D} \mathrm{BN}$ undergo a $(4 \times 4)$ reconstruction and have a band gap smaller than the parent $2 \mathrm{D}$ BN. Oxygen covered 2D BN is found to be a FM, small band gap semiconductor, which may display high spin polarization under bias voltage. If the interaction between adatom and 2D $\mathrm{BN}$ is weak, the electronic structure can be viewed as the 
TABLE IV. Substitution energy, $E_{s}$; magnetic moment per $(4 \times 4)$ supercell, $\mu$; the height of the substituting atom from $2 \mathrm{D} \mathrm{BN}$ monolayer, $h$; the distance from the nearest $\mathrm{B}$ or $\mathrm{N}$ atom, $d_{\mathrm{B} \vee \mathrm{N}}$; charge transferred to substituting atom (Ref. 27), $\Delta \rho$; and energies of relevant localized states in the band gap, $E_{n}$. $(\mathrm{F} \uparrow)$ indicates that the corresponding spin-up state is full. $(\mathrm{E} \downarrow)$ indicates unoccupied spin-down state. If no spin direction is indicated, that state is nonmagnetic.

\begin{tabular}{|c|c|c|c|c|c|c|}
\hline & \multicolumn{3}{|c|}{$\mathrm{B} \rightarrow$} & \multicolumn{3}{|c|}{$\mathrm{N} \rightarrow$} \\
\hline & $\mathrm{Al}$ & $\mathrm{Be}$ & $\mathrm{C}$ & $\mathrm{C}$ & $\mathrm{O}$ & $\mathrm{P}$ \\
\hline $\mathrm{E}_{s}(\mathrm{eV})$ & 5.47 & 5.23 & 5.47 & 3.42 & 6.51 & 3.77 \\
\hline$\mu\left(\mu_{B}\right)$ & NM & NM & 1.00 & 1.00 & 1.00 & NM \\
\hline$h(\AA)$ & 0.52 & 0.01 & 0.01 & 0.00 & 0.00 & 1.42 \\
\hline$d_{\mathrm{B \vee N}}$ & 1.71 & 1.56 & 1.41 & 1.52 & 1.50 & 1.88 \\
\hline$\Delta \rho$ & 2.28 & 1.60 & 1.15 & -1.89 & -1.51 & -0.47 \\
\hline $\mathrm{E}_{1}$ & $2.76(\mathrm{E})$ & $-2.29(\mathrm{~F})$ & $3.50(\mathrm{~F} \uparrow)$ & $-1.69(\mathrm{~F} \uparrow \downarrow)$ & $-2.45(\mathrm{~F} \uparrow)$ & $-0.76(\mathrm{~F})$ \\
\hline $\mathrm{E}_{2}$ & $3.02(\mathrm{E})$ & $-0.83(\mathrm{~F})$ & $4.22(\mathrm{E} \downarrow)$ & $-0.69(\mathrm{~F} \uparrow \downarrow)$ & $-2.16(\mathrm{~F} \downarrow)$ & $-0.07(\mathrm{~F})$ \\
\hline $\mathrm{E}_{3}$ & $4.22(\mathrm{E})$ & $4.38(\mathrm{E})$ & & $0.17(\mathrm{~F} \uparrow)$ & & $4.20(\mathrm{E})$ \\
\hline $\mathrm{E}_{4}$ & $6.26(\mathrm{E})$ & & & $1.07(\mathrm{E} \uparrow)$ & & \\
\hline
\end{tabular}

combination of electronic band structure of 2D BN monolayer and adatom monolayer. $\mathrm{Si}$ and $\mathrm{Fe}$ are weakly bound to $2 \mathrm{D} \mathrm{BN}$ and change the wide band gap of $2 \mathrm{D} \mathrm{BN}$ to a halfmetal.

At low coverage corresponding to $\Theta=1 / 32$, the large distance between adatoms hinders any significant interaction between them. This situation is taken to mimic a single, isolated atom adsorption to 2D $\mathrm{BN}$, which gives rise to localized states in the wide band gap of 2D BN honeycomb structure. This conjecture is confirmed by examining the adsorption of single $\mathrm{C}, \mathrm{O}, \mathrm{Sc}, \mathrm{Ti}$, and $\mathrm{Pt}$ to $(8 \times 8)$ supercell resulting in $\sim 20 \AA$ adatom-adatom distance. In this respect, the adsorbed adatoms at $\Theta=1 / 32$ act as dopants of $2 \mathrm{D} B N$. In a few cases the adsorption site and magnetic state undergo a change by going from the high, $\Theta=1 / 8$ to low coverage, $\Theta=1 / 32$.

Not only adatoms, but also substitution of foreign atoms for $\mathrm{B}$ or $\mathrm{N}$ in the honeycomb structure give rise to localized impurity states in the gap, which attribute interesting electronic properties to the system. In particular, the substitution of $\mathrm{C}$ for $\mathrm{B}$ yields an excess charge and gives rise to two donor states near the edge of conduction band, the lower lying spin-up band being full. In contrast, the substitution of $\mathrm{C}$ for $\mathrm{N}$ yields a single-electron deficiency and gives rise to two acceptor states above the top of valence band, the lower lying spin-up state being full. In both cases, the unpaired spins of $\mathrm{C}$ atom give rise to magnetic moment $\mu=1 \mu_{B}$.

In conclusion, while $2 \mathrm{D} \mathrm{BN}$ is a mechanically stiff and nonmagnetic wide band gap semiconductor, its band gap can be engineered through adatom decoration. In specific cases 2D BN attains magnetic properties and becomes metallic. Foreign atoms adsorbing at low coverage or exchanging with $\mathrm{B}$ or $\mathrm{N}$ atoms give rise to donorlike or acceptorlike states in band gap. At the end, the material achieves interesting properties. Some of these properties can be exploited in future applications.

\section{ACKNOWLEDGMENTS}

Part of computational resources have been provided through a grant (Grant No. 2-024-2007) by the National Center for High Performance Computing, Istanbul Technical University. We thank the DEISA Consortium (www.deisa.eu), funded through the EU FP7 under Project No. RI222919, for support within the DEISA Extreme Computing Initiative. S.C. acknowledges partial support from TUBA, Academy of Science of Turkey. Authors thank Ethem Aktürk for helpful discussions.

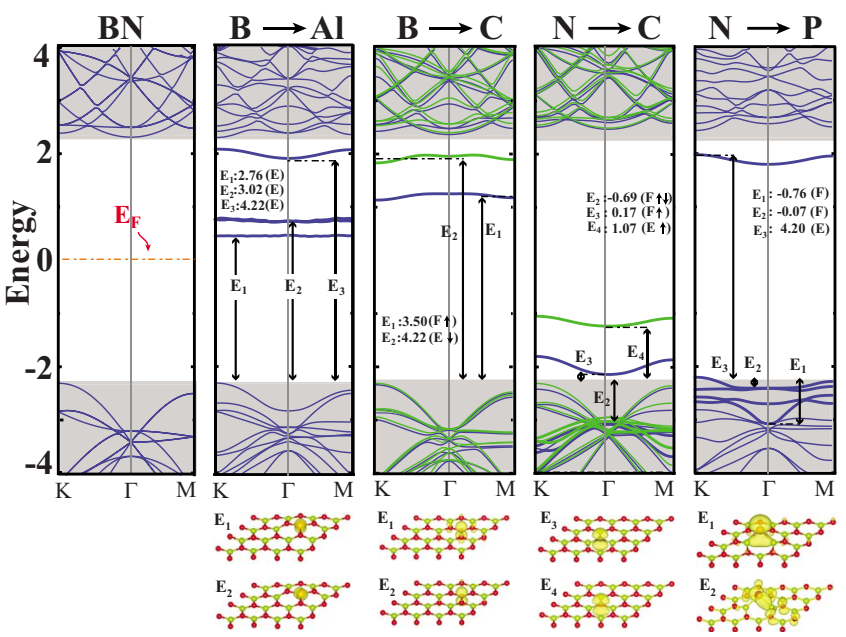

FIG. 6. (Color online) Schematic energy-level diagram of $\mathrm{Al}$ and $\mathrm{C}$ substituting $\mathrm{B}$; and $\mathrm{C}$ and $\mathrm{P}$ substituting $\mathrm{N}$ in a $(4 \times 4)$ supercell of $2 \mathrm{D} \mathrm{BN}$. The light/gray shaded region in the background is the valence and conduction-band continuum of $2 \mathrm{D} \mathrm{BN}$. Zero of energy is set to the Fermi level of bare 2D BN. Spin-up bands are indicated with dark/blue lines whereas spin-down bands are light/ green. Thick solid bands (levels) are states localized at the substituted atom and nearest atoms of 2D BN. Thin solid bands stand for the delocalized states. The energies of some of the localized states with respect to the valence band edge are shown in units of $\mathrm{eV}$. Charge density isosurfaces of some of the relevant states are shown below the corresponding band diagram. The isosurface value is taken as $7 \times 10^{-5}$ electrons $/ \AA^{3}$. 
*ciraci@fen.bilkent.edu.tr

${ }^{1}$ P. F. McMillan, Nature Mater. 1, 19 (2002).

${ }^{2}$ Z. Pan, H. Sun, Y. Zhang, and C. Chen, Phys. Rev. Lett. 102, 055503 (2009).

${ }^{3}$ K. Miyoshi, D. H. Buckley, J. J. Pouch, S. A. Alterovitz, and E. Sliney, Surf. Coat. Technol. 33, 221 (1987).

${ }^{4}$ N. G. Chopra, R. J. Luyken, K. Cherrey, V. H. Crespi, M. K. Cohen, S. G. Louie, and A. Zettl, Science 269, 966 (1995).

${ }^{5}$ R. Arenal, M. Kociak, and N. J. Zaluzec, Appl. Phys. Lett. 90, 204105 (2007).

${ }^{6}$ W. Auwärter, H. U. Suter, H. Sachdev, and T. Greber, Chem. Mater. 16, 343 (2004).

${ }^{7}$ M. Morscher, M. Corso, T. Greber, and J. Osterwalder, Surf. Sci. 600, 3280 (2006).

${ }^{8}$ W. Q. Han, L. Wu, Y. Zhu, K. Watanabe, and T. Taniguchi, Appl. Phys. Lett. 93, 223103 (2008).

${ }^{9}$ D. Pacile, J. C. Meyer, C. O. Girit, and A. Zettl, Appl. Phys. Lett. 92, 133107 (2008).

${ }^{10}$ K. S. Novoselov, D. Jiang, F. Schedin, T. J. Booth, V. V. Khotkevich, S. V. Morozov, and A. K. Geim, Proc. Natl. Acad. Sci. U.S.A. 102, 10451 (2005).

${ }^{11}$ C. Jin, F. Lin, K. Suenaga, and S. Iijima, Phys. Rev. Lett. 102, 195505 (2009).

${ }^{12}$ L. Ci, L. Song, C. Jin, D. Jariwala, D. Wu, Y. Li, A. Srivastava, Z. F. Wang, K. Storr, L. Balicas, F. Liu, and P. M. Ajayan, Nature Mater. 9, 430 (2010).

${ }^{13}$ M. Topsakal, E. Aktürk, and S. Ciraci, Phys. Rev. B 79, 115442 (2009).

${ }^{14}$ H. Şahin, S. Cahangirov, M. Topsakal, E. Bekaroglu, E. Aktürk, R. T. Senger, and S. Ciraci, Phys. Rev. B 80, 155453 (2009).

${ }^{15}$ K. Nose, H. Oba, and T. Yoshida, Appl. Phys. Lett. 89, 112124 (2006).

${ }^{16}$ N. S. Venkataramanan, M. Khazaei, R. Sahara, H. Mizuseki, and Y. Kawazoe, Chem. Phys. 359, 173 (2009).

${ }^{17}$ J. Zhang, S. Wang, X. Du, K. Xu, and V. Ji, J. Phys. Chem. C 113, 17745 (2009).
${ }^{18}$ A. Zobelli, C. P. Ewels, A. Gloter, and G. Seifert, Phys. Rev. B 75, 094104 (2007).

${ }^{19}$ W. Orellana and H. Chacham, Phys. Rev. B 63, 125205 (2001).

${ }^{20}$ M. C. Payne, M. P. Teter, D. C. Allen, T. A. Arias, and J. D. Joannopoulos, Rev. Mod. Phys. 64, 1045 (1992).

${ }^{21}$ Numerical computations have been carried out by using VASP software: G. Kresse and J. Hafner, Phys. Rev. B 47, 558 (1993); G. Kresse and J. Furthmüller, ibid. 54, 11169 (1996).

${ }^{22}$ W. Kohn and L. J. Sham, Phys. Rev. 140, A1133 (1965); P. Hohenberg and W. Kohn, Phys. Rev. 136, B864 (1964).

${ }^{23}$ G. Kresse and D. Joubert, Phys. Rev. B 59, 1758 (1999).

${ }^{24}$ J. P. Perdew, J. A. Chevary, S. H. Vosko, K. A. Jackson, M. R. Pederson, D. J. Singh, and C. Fiolhais, Phys. Rev. B 46, 6671 (1992).

${ }^{25}$ M. Methfessel and A. T. Paxton, Phys. Rev. B 40, 3616 (1989).

${ }^{26}$ H. J. Monkhorst and J. D. Pack, Phys. Rev. B 13, 5188 (1976).

${ }^{27} \mathrm{G}$. Henkelman, A. Arnaldsson, and H. Jonsson, Comput. Mater. Sci. 36, 354 (2006).

${ }^{28}$ C. Kittel, Introduction to Solid State Physics, 7th ed. (Wiley, New York, 1996).

${ }^{29}$ When treated in the $(2 \times 2)$ supercell at $\Theta=1 / 8, \mathrm{C}$ adatoms attain net magnetic moment of $2 \mu_{B}$ per $(2 \times 2)$ supercell. This configuration is, however, $0.3 \mathrm{eV}$ per adatom higher (energetically less favorable) than those reconstructed in the $(4 \times 4)$ supercell. Similarly, for $\Theta=1 / 8 \mathrm{~B}$ treated in $(2 \times 2)$ supercell, binding at the BR site, attain magnetic moment of $1 \mu_{B}$, but becomes $0.12 \mathrm{eV}$ per adatom less favorable relative to the reconstructed structure.

${ }^{30}$ The excited state of AFM ground state of Sc, Ti, and V is the FM state with the magnetic moment of $0.45 \mu_{B}, 3.51 \mu_{B}$, and $4.80 \mu_{B}$; and with the energies of $30 \mathrm{meV}, 50 \mathrm{meV}$, and $20 \mathrm{meV}$ above the ground state, respectively.

${ }^{31}$ The optimized lattice parameters can be different for different adatoms and for different coverages.

${ }^{32}$ C. Ling and D. S. Sholl, Phys. Rev. B 80, 214202 (2009).

${ }^{33}$ R.-F. Liu and C. Cheng, Phys. Rev. B 81, 104407 (2010). 